(C2007 IEEE. Personal use of this material is permitted. However, permission to reprint/republish this material for advertising or promotional purposes or for creating new collective works for resale or redistribution to servers or lists, or to reuse any copyrighted component of this work in other works must be obtained from the IEEE. 


\title{
Harmonic Power Flow Calculations for a Large Power System with Multiple Nonlinear Loads Using Decoupled Approach
}

\author{
A. Ulinuha, M.A.S. Masoum, Senior Member, IEEE, and S.M. Islam, Senior Member, IEEE
}

\begin{abstract}
Power flow calculation is normally carried out for fundamental frequency. Due to the extensive use of nonlinear loads, harmonic frequencies are present and need to be also considered. Unfortunately, unavoidable complexity and heavy computation burden are often encountered by involving nonlinear loads into the calculation. Multiple nonlinear loads typically employed in the real system will further weigh the computation down. This paper implements a decoupled approach to overcome the problem. The couplings between harmonics are rationally disregarded enabling separate calculations for every harmonic order. This will greatly reduce the complexity level and computation burden. However, the accuracy of this technique is somehow questioned due mainly to the neglected harmonic couplings. The accuracy of the implemented decoupled harmonic power flow (DHPF) algorithm is investigated by simulating the distorted IEEE 18-bus system and comparing the results with those generated by standard packages (e.g., HARMFLOW and ETAP). The implementation is then extended for the IEEE 123bus system including multiple nonlinear loads.
\end{abstract}

Index Terms - Accuracy, decoupled, efficiency, harmonic power flow, multiple nonlinear loads

\section{INTRODUCTION}

$\mathrm{P}^{\mathrm{o}}$ OWER flow calculation is backbone of power system analysis and design. It generates the results that are normally required for further calculation of analysis and design. The calculation is initially performed by formulating the network equation. Node-voltage method, which is the most suitable form for many power system analyses, is commonly used. Mathematically, power flow problem requires solution of simultaneous nonlinear equations and normally employs an iterative method, such as Gauss-Seidel and Newton-Raphson.

The aforementioned calculation is typically carried out by simply considering fundamental frequency. The extensive and ever increasing applications of nonlinear loads such as power electronic devices result in the existence of higher components other than that of fundamental frequency, called harmonics. The nonlinear voltage-current relationship of these devices results in harmonic currents that propagate through the system and produce potentially dangerous harmonic voltages. This

The Authors are with the Department of Electrical and Computer Engineering, Curtin University of Technology, Perth, WA, 6845, Australia, email: agus.ulinuha@postgrad.curtin.edu.au or guslin70@yahoo.com phenomenon has become a major concern for power quality and therefore harmonics must be included in the calculations to predict their effects and to avoid possible severe damages. However, taking harmonics into account will lead the calculations to be very complicated.

Real power systems usually employ a number of nonlinear loads with different $v-i$ characteristics. This results in multiple harmonic injection currents with different orders, magnitudes and phase angles. This will complicate the calculation even further.

Applications of decoupled approach for harmonic power flow calculation including multiple nonlinear loads are presented in this paper. The aim is to exhibit the inclusion of harmonics in power flow calculation with a reasonable computation burden. Due to the accuracy of decoupled harmonic power flow (DHPF) is somehow questioned, the accuracy of the algorithm is therefore verified by comparing its generated results with those generated by HARMFLOW [1] and ETAP [2]. The IEEE 18-bus distorted system [3] with one nonlinear load is simulated for comparison purposes. The application of the approach is then extended for IEEE 18-bus and IEEE 123-bus systems with multiple nonlinear loads. It is shown that the decoupled approach offers a compromise between result accuracy and computation complexity.

\section{HARMONIC POWER FLOW}

Harmonic power flow was initially introduced by Xia and Heydt [1] by involving nonlinear loads in power flow calculation. Conventionally, power flow is formulated on the basis that power sources are system generators and power "sinks" are the loads. Harmonic power flow, on the other hand, is more general in that loads may be the "source" of harmonic energy [4]. The ultimate source is system generators, but harmonic distortion that occurs at bus containing nonlinear load may be considered as a source of harmonic signal. For the system consisting of multiple nonlinear loads harmonic currents are therefore injected by a number of nonlinear loads. In addition to some results normally generated by power flow, harmonic power flow also generates other results that can be used to quantify voltage distortion and to determine whether dangerous resonant problem exists.

The nature of the harmonics (i.e., orders, magnitudes and phases) strongly depends on the nonlinear load involved. 
Therefore, nonlinear load modeling is essential part of harmonic power flow calculation. A particular nonlinear load needs to be specifically modeled. Therefore, a system including different types of nonlinear loads requires specific model for every nonlinear type.

Nonlinear loads can be modeled in time and/or frequency domain [5]. Time domain modeling is based on transient-state analysis while frequency domain modeling uses frequencyscan process to calculate the frequency response of a system. Time domain modeling requires detailed representation of the device that increases the problem complexity resulting in prohibitively long computation time. Therefore, frequency domain methods are commonly used for harmonic analysis to reduce the computation time. For nonlinear loads that can be presented as voltage-independent current sources, frequency domain model can be applied for harmonic power flow analysis [6].

Harmonic power flow calculations can generally be classified into coupled and decoupled methods. Coupled approach solves all harmonic orders simultaneously. This approach has good accuracy but leads to a greater computational cost as the problem becomes quite complicated. It also requires exact formulation of nonlinear loads that is sometimes practically unavailable resulting in limited applications [7]. On the other hand, decoupled approach assumes that the coupling between harmonic orders can be rationally disregarded and, as a result, the calculation can be separately carried out for every harmonic order. Therefore, this approach requires less computational charge. In addition, since nonlinear loads are modeled with harmonic current or voltage sources, it is very easy to include them in the calculations using measured non-sinusoidal current and/or voltage waveforms. For the system serving multiple nonlinear loads, the different type of nonlinear loads may be easily included in calculations by simultaneously considering harmonic currents injected by the nonlinear loads. Although the decoupled approach is not as accurate as the coupled technique, it offers a compromise between computational complexity and result accuracy.

\section{A. Decoupled Approach for Harmonic Power Flow}

At the fundamental frequency, system is modeled using the conventional approach where the admittance of line section between bus $i$ and bus $i+1$ is expressed as follows.

$$
y_{i, i+1}=\frac{1}{R_{i, i+1}+j X_{i, i+1}}
$$

Where $R_{i, i+1}$ and $X_{i, i+1}$ are the respective resistance and inductance of line section between bus $i$ and $i+1$. The magnitude and phase angle of bus voltage is then calculated using the following mismatch equations [8-10].

$$
\begin{gathered}
P_{i}-\sum_{j=i-1}^{i+1}\left|Y_{j i}^{1}\left\|V_{j}^{1}\right\| V_{i}^{1}\right| \cos \left(\delta_{i}^{1}-\delta_{j}^{1}-\theta_{j i}^{1}\right)=0 \\
Q_{i}-\sum_{j=i-1}^{i+1}\left|Y_{j i}^{1}\left\|V_{j}^{1}\right\| V_{i}^{1}\right| \sin \left(\delta_{i}^{1}-\delta_{j}^{1}-\theta_{j i}^{1}\right)=0
\end{gathered}
$$

Where

$$
Y_{j i}^{1}=\left|Y_{j i}^{1}\right| \angle \theta_{j i}^{1}= \begin{cases}-y_{j i}^{1}, & \text { if } j \neq i \\ y_{i-1, i}^{1}+y_{i+1, i}^{1}+y_{c i}^{1}, & \text { if } j=i\end{cases}
$$

$V_{i}^{1}$ and $y_{c i}^{1}$ are the respective fundamental voltage and admittance of shunt capacitor at bus $i$, while $P_{i}$ and $Q_{i}$ are the respective total (linear and nonlinear) active and reactive powers at bus $i$. Power loss in the line section between bus $i$ and $i+1$ may be calculated by the following equation.

$$
P_{\text {loss }(i, i+1)}^{1}=R_{i, i+1}\left(\left|V_{i, i+1}^{1}-V_{i}^{1} \| y_{i, i+1}^{1}\right|\right)^{2}
$$

At harmonic frequencies, power system is modeled as combination of passive elements and current sources [8]. The system can then be considered as a passive element with multiple harmonic injection currents. The generalized model is suggested for a linear load, which is composed by a resistance in parallel with an inductance to account for the respective active and reactive loads at fundamental frequency. Nonlinear loads, in general, are considered as ideal harmonic current sources that generate harmonic currents and inject them into the system [11]. The admittance-matrix-based harmonic power flow is the most widely used method as it is based on the frequency-scan process [12]. In this approach, admittance of system components will vary with the harmonic order. If skin effect is ignored at higher frequencies, the resulting $h^{\text {th }}$ harmonic frequency load admittance, shunt capacitor admittance and feeder admittance are respectively given by the following equations [8-10, 13-16].

$$
\begin{gathered}
y_{l i}^{h}=\frac{P_{l i}}{\left|V_{i}^{1}\right|^{2}}-j \frac{Q_{l i}}{h\left|V_{i}^{1}\right|^{2}} \\
y_{c i}^{h}=h y_{c i}^{1} \\
y_{i, i+1}^{h}=\frac{1}{R_{i, i+1}+j h X_{i, i+1}}
\end{gathered}
$$

Where $P_{l i}$ and $Q_{l i}$ are the respective active and reactive linear loads at bus $i$. The nonlinear load is treated as harmonic current sources and the $h^{\text {th }}$ harmonic current injected at bus $i$ introduced by the nonlinear load with real power $P_{n}$ and reactive power $Q_{n}$ is derived as follows:

$$
\begin{gathered}
I_{i}^{1}=\left[\left(P_{n i}+j Q_{n i}\right) / V_{i}^{1}\right]^{*} \\
I_{i}^{h}=C(h) I_{i}^{1}
\end{gathered}
$$

Where $I_{i}^{1}$ is the fundamental current and $I_{i}^{h}$ is the $h^{\text {th }}$ harmonic current determined by $C(h)$, which is the ratio of the $h^{\text {th }}$ harmonic to the fundamental current. $C(h)$ can be obtained by field test and Fourier analysis for all customers along the distribution feeder $[8,10,16]$.

For decoupled harmonic power flow calculation, loop equations are written at each harmonic frequency of interest. Each loop is formed including the source nodes. After modifying admittance matrix and the associated harmonic currents, the harmonic load flow problem can then be calculated using the following equation $[12,15,16]$. 


$$
Y^{h} V^{h}=I^{h}
$$

At any bus $i$, the rms voltage is defined as:

$$
\left|V_{i}\right|=\left(\sum_{h=1}^{H}\left|V_{i}^{h}\right|^{2}\right)^{1 / 2}
$$

Where $H$ is the maximum harmonic orders considered. After solving load flow for different harmonic orders, the distortion of voltage indicated by total harmonic distortion at bus $i$ $\left(\mathrm{THD}_{v i}\right)$ is expressed by the following equation.

$$
T H D_{v i}(\%)=\left[\frac{\left(\sum_{n \neq 1}^{H}\left|V_{i}^{h}\right|^{2}\right)^{1 / 2}}{\left|V_{i}^{1}\right|}\right] \times 100 \%
$$

At the $h^{\text {th }}$ harmonic frequency, real power loss in the line section between buses $i$ and $i+1$ is expressed below [9, 10, $16]$.

$$
P_{l o s s(i, i+1)}^{h}=R_{i, i+1}\left(\left|V_{i, i+1}^{h}-V_{i}^{h}\right|\left|y_{i, i+1}^{h}\right|\right)^{2}
$$

The total power loss including the loss at fundamental frequency is given by the following equation.

$$
P_{\text {loss }}^{h}=\sum_{h=1}^{H}\left(\sum_{i=1}^{m} P_{l o s s(i, i+1)}^{h}\right)
$$

Where $m$ is number of bus. The computation procedure of the proposed approach is given by Fig. 1 .

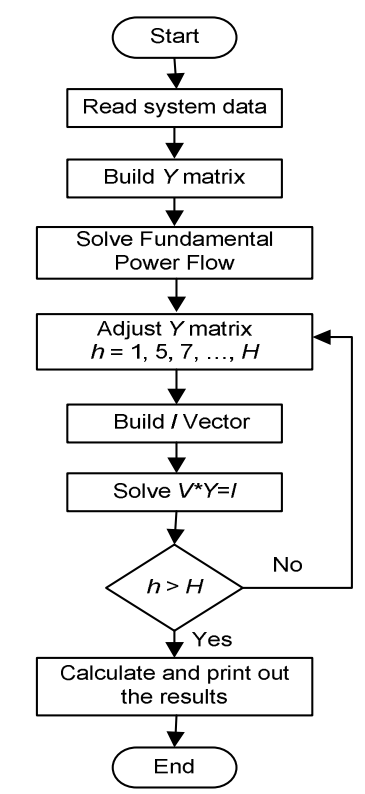

Fig. 1. Decoupled Harmonic Power Flow Calculation

\section{RESULT AND DISCUSSION}

\section{A. Results}

For verification purpose, the decoupled approach is initially implemented for the IEEE 18-bus distorted system [3] of Fig. 2 considering only one nonlinear load (six-pulse 1, $3 \mathrm{MW}$,
2.26 MVAR) installed at bus 5. The nonlinear is modeled as current sources with harmonic contents listed in Appendix. The generated results are then compared with those generated by standard packages (HARMFLOW [1] and ETAP [2]). The simulation is coded using MATLAB version 7.0.1 R14 and is run in a desktop PC with Pentium 4 Intel $3.0 \mathrm{GHz}$ processor and 512 MB RAM.

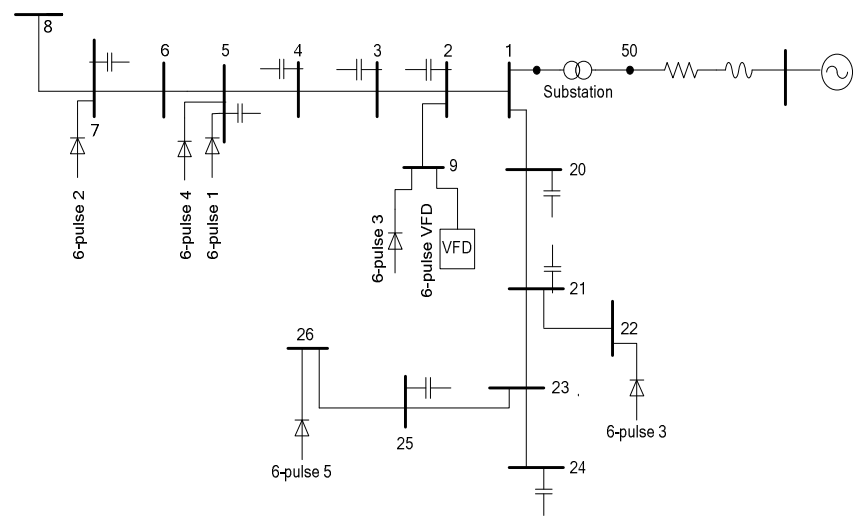

Fig. 2. The simulated IEEE 18-bus distorted distribution system [3]

The comparisons of the results generated by decoupled approach with those generated by the HARMFLOW and ETAP are separately performed due to the harmonic orders considered in HARMFLOW are different with those considered in ETAP. The comparisons with HARMFLOW are indicated in Fig. 3 (rms Voltage) and Fig. 4 (THD) while the comparisons with ETAP are shown in Fig. 5 (rms Voltage) and Fig. 6 (THD), respectively. As predicted, there are some slight differences at some buses due to the neglected harmonic couplings in DHPF. The comparisons are summarized in Table 1 demonstrating the fine accuracy of the implemented approach.

TABLE 1

AVERAGE DEVIATION OF THE RESUlTS GENERATED BY DECOUPLED APPROACH AND HARMFLOW [1] AND ETAP [2]

\begin{tabular}{|c|c|c|}
\hline \multirow{2}{*}{ APPROACH AND HARMFLOW [1] AND ETAP [2] } \\
\cline { 2 - 3 } & \multicolumn{2}{|c|}{ Average deviation (\%) with } \\
\hline Vfund & 0.005870 & 0.002134 \\
\hline Vrms & 0.027797 & 0.026716 \\
\hline THDv & 0.572418 & 0.499733 \\
\hline
\end{tabular}

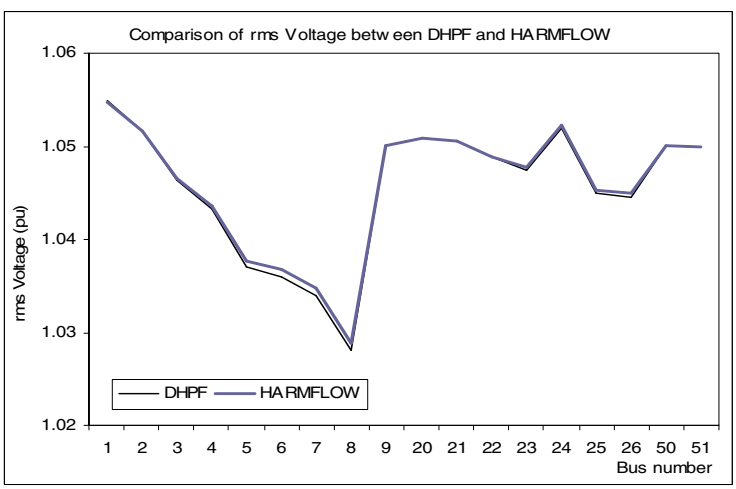

Fig. 3. rms Voltage comparison between DHPF and HARMFLOW 


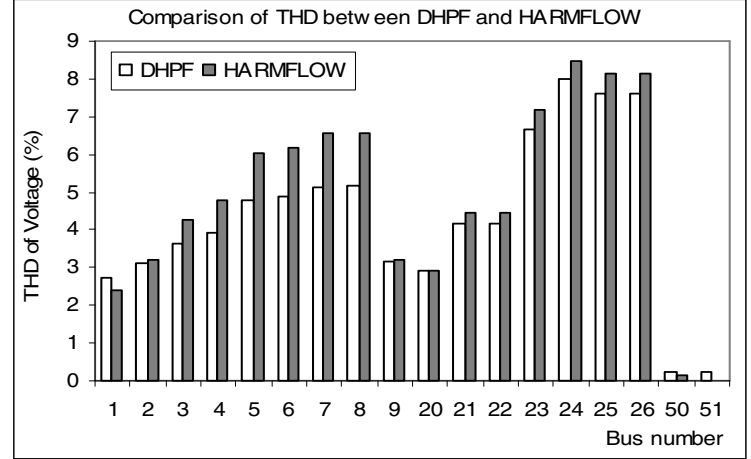

Fig. 4. THD comparison between DHPF and HARMFLOW

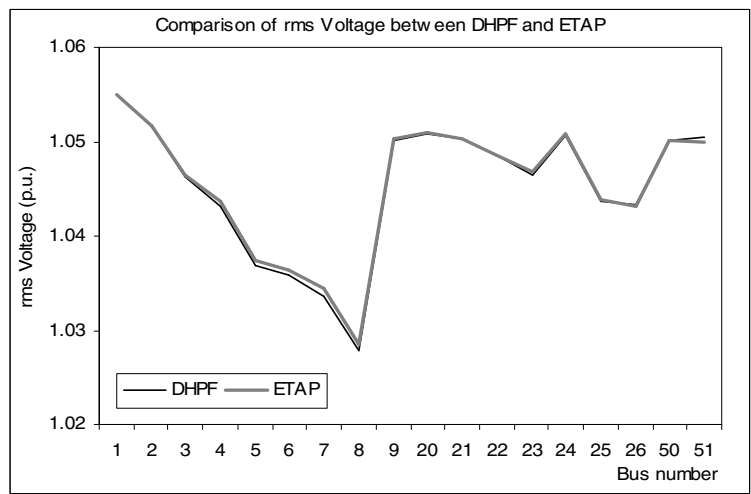

Fig. 5. rms Voltage comparison between DHPF and ETAP

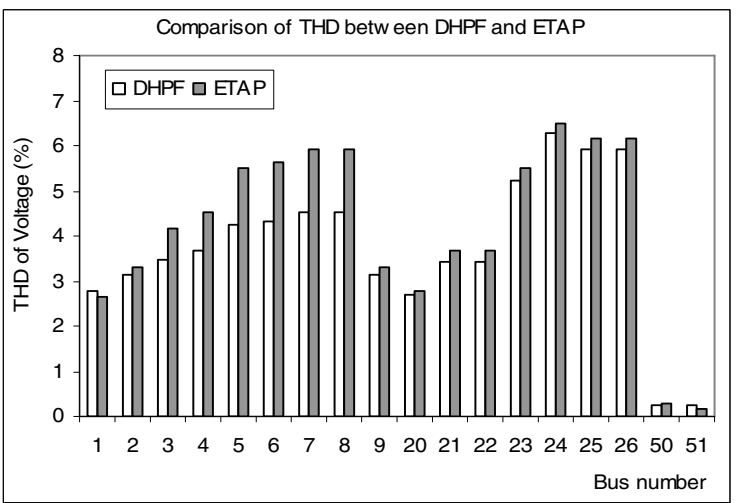

Fig. 6. THD comparison between DHPF and ETAP

The implementation of decoupled approach is extended for IEEE 18-bus system of Fig. 2 considering all installed nonlinear loads. The nonlinear loads are indicated in Table 2 and their harmonic contents are given in Appendix. Computationally, the voltage calculations at harmonic frequencies are carried out by initially forming vector of injection currents and finding the harmonic voltages by solving Eq. (11). The generated results including fundamental and rms voltages for the simulated system are given in Fig. 7 illustrating the increment of voltage caused by harmonic injection currents. The THD of voltage is shown in Fig. 8 showing the spreading of harmonic distortion among of buses due to the distributed nonlinear loads.
TABLE II

NONLINEAR LOADS USED IN IEEE 18-BUS SYSTEM

\begin{tabular}{|c|l|c|c|}
\hline \multicolumn{2}{|c|}{ Nonlinear Load } & \multicolumn{2}{c|}{ Power } \\
\hline Bus & \multicolumn{1}{|c|}{ Name } & MW & MVAR \\
\hline 5 & six-pulse 1 & 1.20 & 0.75 \\
\hline 5 & six-pulse 4 & 0.75 & 0.50 \\
\hline 7 & six-pulse 2 & 1.00 & 0.60 \\
\hline 9 & six-pulse VFD & 1.50 & 0.75 \\
\hline 9 & Six-pulse 3 & 1.50 & 0.75 \\
\hline 22 & six-pulse 3 & 0.80 & 0.50 \\
\hline 26 & six-pulse 5 & 1.00 & 0.60 \\
\hline
\end{tabular}

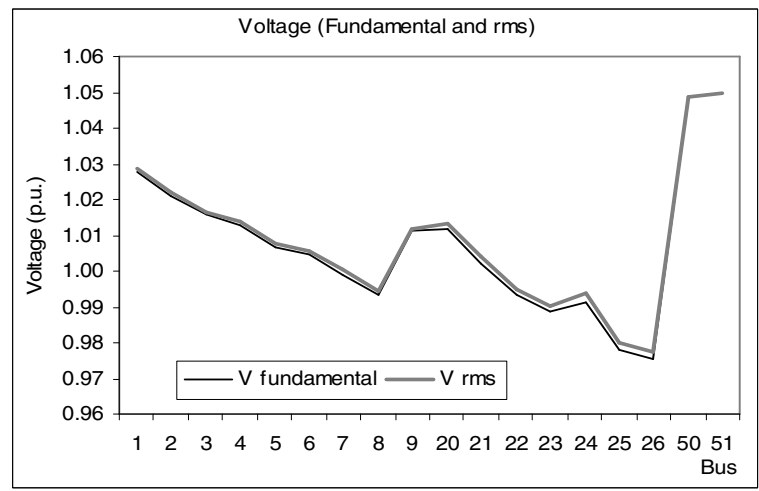

Fig. 7. rms Voltage of the distorted IEEE 18-bus system with multiple nonlinear loads generated by DHPF

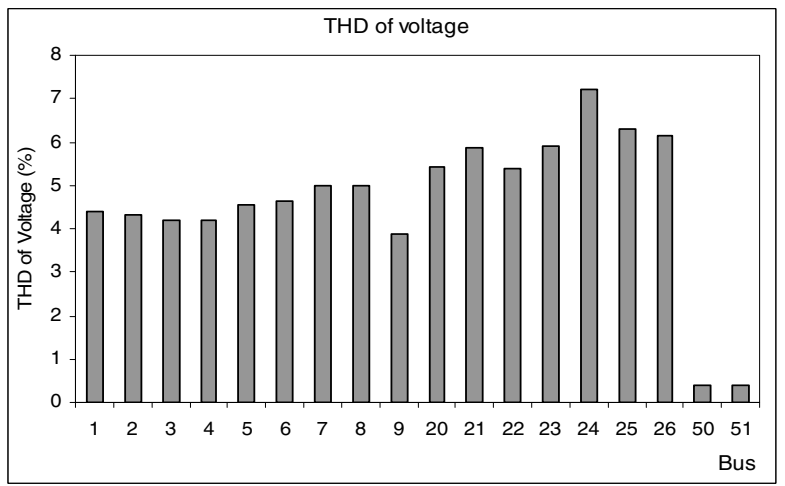

Fig. 8. THD of voltage of the distorted IEEE 18-bus system with multiple nonlinear loads generated by DHPF

To exhibit the ability of decoupled approach for handling large distorted system, the approach is now implemented for IEEE 123-bus distribution system [17]. The simulated system including 12 nonlinear loads is shown in Fig. 9. The installed nonlinear loads data are given in Table 3 and their harmonic contents are indicated in Appendix. The generated results including fundamental and rms voltages as well as THD of voltage are respectively shown in Fig. 10 and Fig. 11.

Successful application of DHPF for the IEEE 123-bus with multiple nonlinear loads confirms the ability of the approach simulating large distorted system without any convergence problem. The approach is also able accommodating multiple nonlinear loads necessary for practical calculation. 


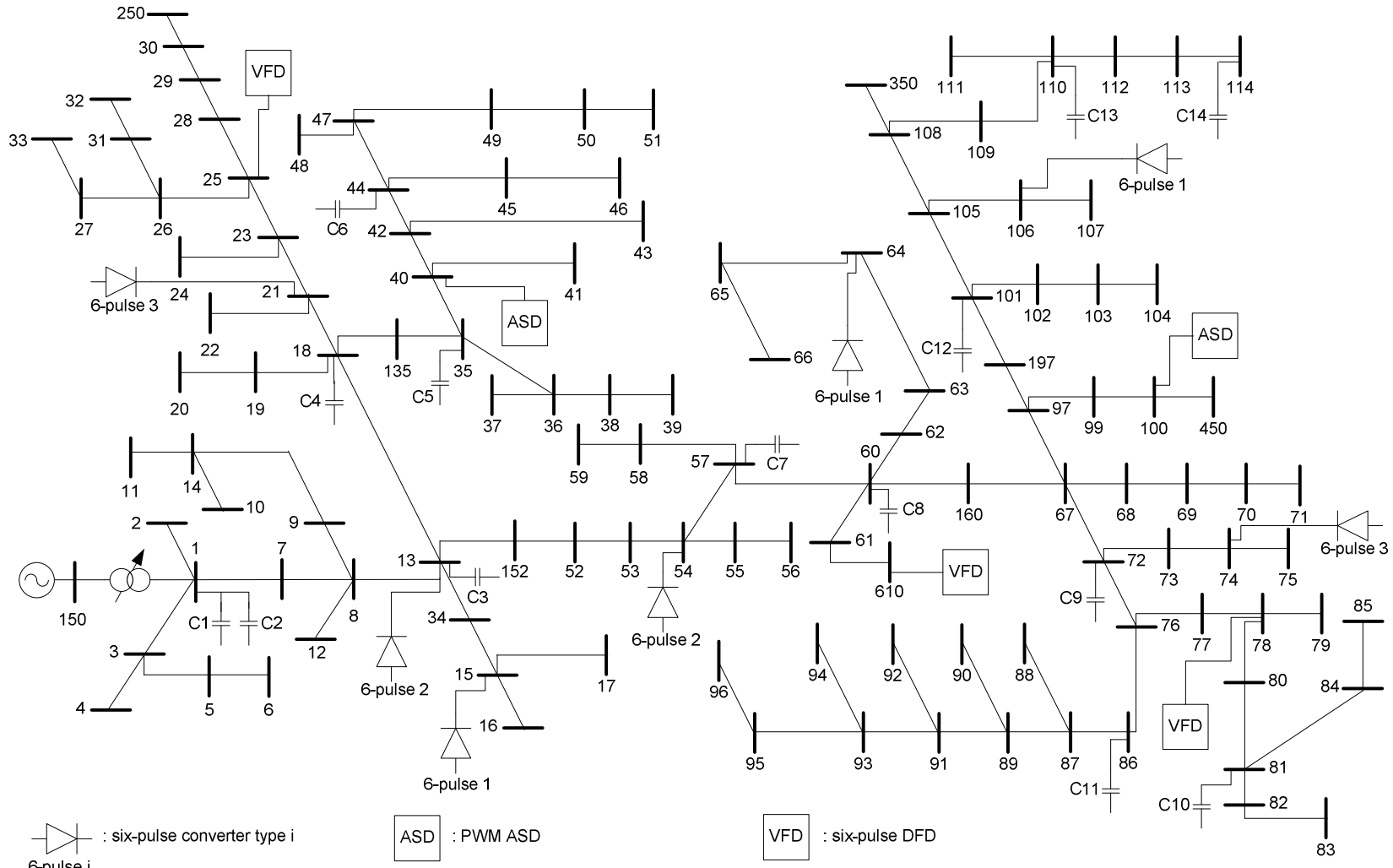

Fig. 9. The IEEE 123-bus distorted distribution system used for simulation

TABLE III

NONLINEAR LOADS USED IN IEEE 123-BUS SYSTEM

\begin{tabular}{|c|l|c|c|}
\hline \multicolumn{2}{|c|}{ Nonlinear Load } & \multicolumn{2}{c|}{ Power } \\
\hline Bus & \multicolumn{1}{|c|}{ Name } & MW & MVAR \\
\hline 13 & Six-pulse2 & 0.0247 & 0.0176 \\
\hline 15 & Six-pulse1 & 0.0315 & 0.0196 \\
\hline 21 & Six-pulse4 & 0.0383 & 0.0264 \\
\hline 25 & six-pulse VFD & 0.0354 & 0.0179 \\
\hline 40 & PWM-ASD & 0.0474 & 0.0293 \\
\hline 54 & Six-pulse2 & 0.0382 & 0.0151 \\
\hline 64 & Six-pulse1 & 0.0213 & 0.0093 \\
\hline 74 & Six-pulse4 & 0.0257 & 0.0172 \\
\hline 78 & six-pulse VFD & 0.0384 & 0.0142 \\
\hline 100 & PWM-ASD & 0.0247 & 0.0184 \\
\hline 106 & Six-pulse1 & 0.0192 & 0.0094 \\
\hline 610 & six-pulse VFD & 0.0385 & 0.0195 \\
\hline
\end{tabular}

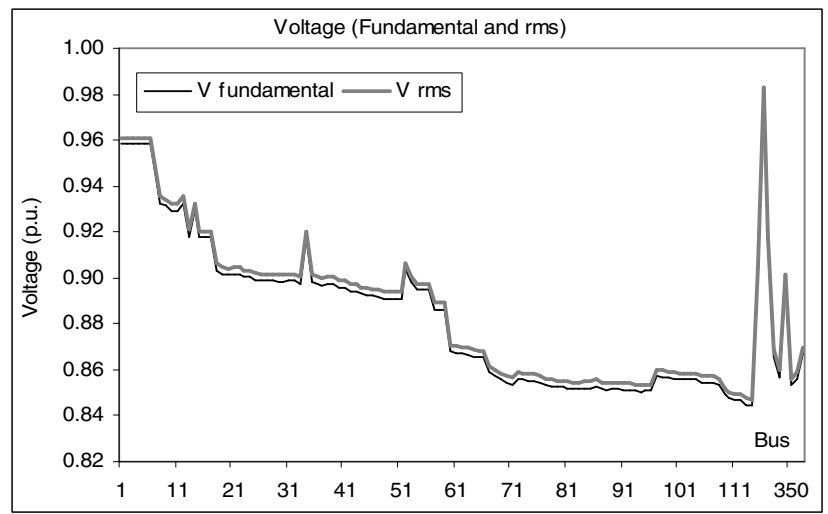

Fig. 10. rms Voltage of the distorted IEEE 123-bus system with multiple nonlinear loads generated by DHPF

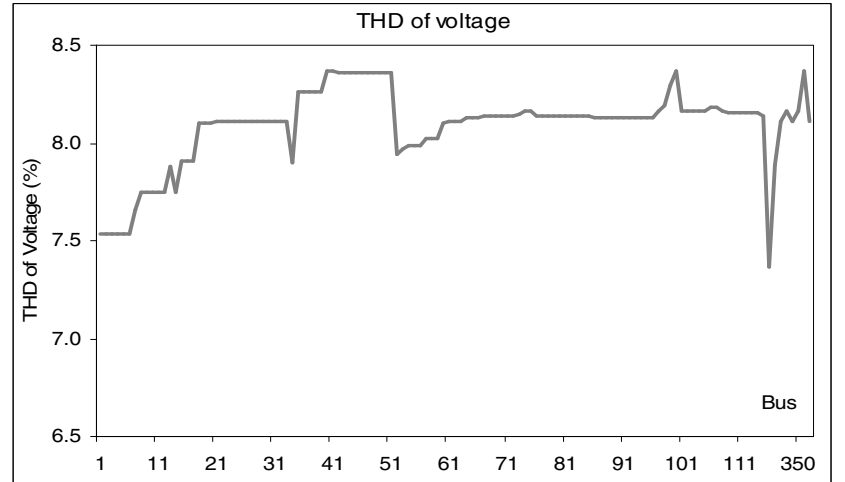

Fig. 11. THD of voltage of the distorted IEEE 123-bus system with multiple nonlinear loads generated by DHPF

\section{B. Discussion}

In decoupled approach, harmonic voltage calculations are separately performed for every harmonic order. This approach is therefore simple and can be used to simulate large systems without convergence difficulties. Most harmonic power flow algorithms are not capable for simulating large systems (e.g., with hundreds of buses). Representation of nonlinear load as current source leads to extensive applications as the approach accepts the nonlinear load data obtained from measurements. Coupled approaches require exact models of nonlinear loads that are not always available. Furthermore, DHPF has less computational burden resulting in less computation time. Furthermore, DHPF has less computational burden resulting in less computation time. 


\section{CONCLUSION}

Harmonic power flow calculations for distorted systems serving multiple nonlinear loads are carried out using decoupled approach. Main conclusions are:

- Decoupled approach offers compromise between results accuracy and calculations complexity.

- The approach is capable of simulating large system serving multiple nonlinear loads with different $v-i$ characteristics.

- Modeling of nonlinear loads as current sources lead to extensive application as it accepts real measurement data.

\section{APPENDIX}

Harmonic contents of nonlinear loads used in this paper are given in Table A.1.

TABLE A. 1

HARMONIC CONTENTS OF NONLINEAR LOADS USED IN THIS PAPER

\begin{tabular}{|c|c|c|c|c|c|c|c|c|}
\hline \multirow{3}{*}{$\stackrel{\bar{d}}{\overrightarrow{0}}$} & \multicolumn{8}{|c|}{ Nonlinear Loads } \\
\hline & \multicolumn{2}{|c|}{ Six-pulse1 } & \multicolumn{2}{|c|}{ Six-pulse2 } & \multicolumn{2}{|c|}{ Six-pulse3 } & \multicolumn{2}{|c|}{ Six-pulse 4} \\
\hline & mag & deg & mag & deg & mag & deg & mag & deg \\
\hline 1 & 100 & 0 & 100 & 0 & 100 & 0 & 100 & 0 \\
\hline 5 & 20 & 0 & 19.1 & 0 & 20 & 0 & 42 & 0 \\
\hline 7 & 14.3 & 0 & 13.1 & 0 & 14.3 & 0 & 14.3 & 0 \\
\hline 11 & 9.1 & 0 & 7.2 & 0 & 9.1 & 0 & 7.9 & 0 \\
\hline 13 & 7.7 & 0 & 5.6 & 0 & 0 & 0 & 3.2 & 0 \\
\hline 17 & 5.9 & 0 & 3.3 & 0 & 0 & 0 & 3.7 & 0 \\
\hline 19 & 5.3 & 0 & 2.4 & 0 & 0 & 0 & 2.3 & 0 \\
\hline 23 & 4.3 & 0 & 1.2 & 0 & 0 & 0 & 2.3 & 0 \\
\hline 25 & 4 & 0 & 0.8 & 0 & 0 & 0 & 1.4 & 0 \\
\hline 29 & 3.4 & 0 & 0.2 & 0 & 0 & 0 & 0 & 0 \\
\hline 31 & 3.2 & 0 & 0.2 & 0 & 0 & 0 & 0 & 0 \\
\hline 35 & 2.8 & 0 & 0.4 & 0 & 0 & 0 & 0 & 0 \\
\hline 37 & 2.7 & 0 & 0.5 & 0 & 0 & 0 & 0 & 0 \\
\hline 41 & 2.4 & 0 & 0.5 & 0 & 0 & 0 & 0 & 0 \\
\hline 43 & 2.3 & 0 & 0.5 & 0 & 0 & 0 & 0 & 0 \\
\hline 47 & 2.1 & 0 & 0.4 & 0 & 0 & 0 & 0 & 0 \\
\hline 49 & 2 & 0 & 0.4 & 0 & 0 & 0 & 0 & 0 \\
\hline
\end{tabular}

TABLE A.1 (CONTINUED)

HARMONIC CONTENTS OF NONLINEAR LOADS USED IN THIS PAPER

\begin{tabular}{|c|r|r|r|r|r|c|}
\hline \multirow{2}{*}{$\underset{\tilde{\Xi}}{\tilde{\sigma}}$} & \multicolumn{9}{|c|}{ Nonlinear Loads } \\
\cline { 2 - 7 } & \multicolumn{2}{|c|}{ six-pulse 5 } & \multicolumn{2}{c|}{ six-pulse VFD } & \multicolumn{2}{c|}{ PWM-ASD } \\
\cline { 2 - 7 } & mag & deg & mag & deg & mag & deg \\
\hline 1 & 100 & 0 & 100 & 0 & 100 & 0 \\
\hline 5 & 28 & 0 & 23.52 & 111 & 82.8 & -135 \\
\hline 7 & 9 & 0 & 6.08 & 109 & 77.5 & 69 \\
\hline 11 & 9 & 0 & 4.57 & -158 & 46.3 & -62 \\
\hline 13 & 6 & 0 & 4.2 & -178 & 41.2 & 139 \\
\hline 17 & 5 & 0 & 1.8 & -94 & 14.2 & 9 \\
\hline 19 & 4 & 0 & 1.37 & -92 & 9.7 & -155 \\
\hline 23 & 3 & 0 & 0.75 & -70 & 1.5 & -158 \\
\hline 25 & 3 & 0 & 0.56 & -70 & 2.5 & 98 \\
\hline 29 & 0 & 0 & 0.49 & -20 & 0 & 0 \\
\hline 31 & 0 & 0 & 0.54 & 7 & 0 & 0 \\
\hline
\end{tabular}

Note:

mag: Magnitude of harmonic currents with respect to its fundamental value (\%)

deg: Angle of harmonic currents.

\section{REFERENCES}

[1] D. Xia and G. T. Heydt, "Harmonic power flow studies, part I Formulation and Solution," IEEE Trans. on Power Apparatus and System, vol. 101, pp. 1257-1265, June 19821982.

[2] "ETAP PowerStation ", 4.7.0E ed Birtcher Drive, Lake Forest: Operation Technology, Inc., 2002.
[3] W. M. Grady, M. J. Samotyj, and A. H. Noyola, "The application of network objective functions for actively minimizing the impact of voltage harmonics in power systems," IEEE Transactions on Power Delivery, vol. 7, pp. 1379-1386, 1992.

[4] A. Semlyen and M. Shlash, "Principles of modular harmonic power flow methodology," IEE Proceedings-Generation, Transmission and Distribution, vol. 147, pp. 1-6, 2000.

[5] M. A. Moreno Lopez de Saa and J. Usaola Garcia, "Three-phase harmonic load flow in frequency and time domains," IEE Proceedings Electric Power Applications, vol. 150, pp. 295-300, 2003.

[6] Y.-Y. Hong, J.-S. Lin, and C.-H. Liu, "Fuzzy harmonic power flow analyses," in International Conference on Power System Technology, PowerCon, 2000, pp. 121-125.

[7] S. M. Williams, G. T. Brownfield, and J. W. Duffus, "Harmonic propagation on an electric distribution system: field measurements compared with computer simulation," IEEE Transactions on Power Delivery, vol. 8, pp. 547-552, 1993.

[8] H.-C. Chin, "Optimal shunt capacitor allocation by fuzzy dynamic programming," Electric Power Systems Research, vol. 35, pp. 133-139, 1995/11 1995.

[9] Y. Baghzouz and S. Ertem, "Shunt capacitor sizing for radial distribution feeders with distorted substation voltage," IEEE Trans. on Power Delivery, vol. 5, pp. 650-657, Apr. 1990. 1990.

[10] Y. Baghzouz, "Effects of nonlinear loads on optimal capacitor placement in radial feeders," IEEETrans. on Power Delivery, vol. 6, pp. 245-251, Jan. 19911991.

[11] X.-m. Yu, X.-y. Xiong, and Y.-w. Wu, "A PSO-based approach to optimal capacitor placement with harmonic distortion consideration," Electric Power Systems Research, vol. 71, pp. 27-33, 2004/9 2004.

[12] J.-H. Teng and C.-Y. Chang, "Fast harmonic analysis method for unbalanced distribution systems," in Power Engineering Society General Meeting, 2003, IEEE, 2003, p. 1249 Vol. 2.

[13] M. A. S. Masoum, A. Jafarian, M. Ladjevardi, E. F. Fuchs, and W. M. Grady, "Fuzzy approach for optimal placement and sizing of capacitor banks in the presence of harmonics," IEEE Transactions on Power Delivery, vol. 19, pp. 822 - 829, April 20042004.

[14] M. A. S. Masoum, M. Ladjevardi, A. Jafarian, and E. F. Fuchs, "Optimal Placement, Replacement and Sizing of Capacitor Banks in Distorted Distribution Networks by Genetic Algorithms," IEEE Transactions on Power Delivery, vol. 19, pp. 1794-1801, 2004.

[15] T. Ghose and S. K. Goswami, "Effects of unbalances and harmonics on optimal capacitor placement in distribution system," Electric Power Systems Research, vol. 68, pp. 167-173, 2003/2 2003.

[16] T. S. Chung and H. C. Leung, "A genetic algorithm approach in optimal capacitor selection with harmonic distortion considerations," International Journal of Electrical Power \& Energy Systems, vol. 21, pp. 561-569, 1999/11 1999.

[17] W. H. Kersting, "Radial distribution test feeders," IEEE Transactions on Power Systems, vol. 6, pp. 975-985, 1991. 